\title{
ANÁLISIS DE CRITICIDAD Y ÁRBOLES DE DIAGNÓSTICO DE FALLAS PARA TRANSFORMADORES DE POTENCIA
}

\section{CRITICALITY ANALYSIS AND FAULT DIAGNOS IS TREES FOR POWER TRANSFORMERS}

\author{
Ing. Jesus Enrique Salamanca Jaimes*, MSc. Jesus Abelardo Velazco Ochoa** \\ MSc. Eduar Alberto Acevedo Camacho*
}

*Centrales Eléctricas del Norte de Santander S.A. E.S.P.

Subgerencia de subestaciones y líneas. Cúcuta, Colombia.

E-mail: jexuz27@hotmail.com, eduar.acevedo@cens.com.co

**Universidad de Los Andes, Facultad de Ingeniería.

Núcleo Universitario Pedro Rincón Gutiérrez, La Hechicera, Municipio Libertador.

Parroquia Milla. Edificio Facultad de Ingeniería, Nivel 3 Ala Este. Mérida - Venezuela. Tel.: (+58) (274) 240 2930/2931, Fax: (+58) (274) 2402806.

E-mail: javelazco@gmail.com

\begin{abstract}
Resumen: El desarrollo de este artículo está dirigido a plantear el análisis de criticidad como modelo de jerarquización en subsistemas donde intervienen equipos de patio de subestaciones eléctricas propiedad de Centrales Eléctricas del Norte de Santander SA ESP. Este análisis es replicable a otros subsistemas y está basado en la recopilación de información de un grupo de trabajo específico con amplia experiencia en el mantenimiento de los activos objeto de estudio. Además de este análisis, se propone la implementación de árboles de diagnóstico de fallas para transformadores de potencia como herramienta fundamental en el análisis predictivo de fallas en este tipo de activo, cuya operación es fundamental para ofrecer un servicio continuo y de calidad.
\end{abstract}

Palabras clave: Activos, análisis de criticidad, diagnóstico de fallas, mantenimiento.

\begin{abstract}
The development of this article is intended to raise the criticality analysis and hierarchical model into subsystems which involved teams yard substations owned Power Plant of Norte de Santander SA ESP. This analysis is replicable to other subsystems and is based on gathering information of a specific working group with extensive experience in maintaining the assets under consideration. In addition to this analysis, the implementation of troubleshooting trees for power transformers as a fundamental tool in predictive failure analysis on this type of asset is proposed, whose operation is critical to provide continuous quality service.
\end{abstract}

Keywords: Asset criticality analys is, troubleshooting, maintenance.

\section{INTRODUCCIÓN}

La Confiabilidad Operacional como estrategia esencial de la Gestión de Activos, permite definir el desarrollo de procesos de mejora continua que incorporan en forma sistémica y sistemática avanzadas herramientas de diagnóstico, metodologías de análisis y nuevas tecnologías de mantenimiento, para optimizar la planeación, ejecución y control de la producción industrial. (Becerra y García, 2005). 
La gestión de activos es de gran relevancia para las industrias; con la creciente oferta de productos y servicios y la automatización de los procesos, es de suma importancia optimizar los recursos al máximo; el mantenimiento predictivo se basa en técnicas y metodologías que permiten a un activo sea útil durante el $100 \%$ de su vida, sin hacer cambios del mismo o paradas innecesarias; por tal motivo se presenta el análisis de criticidad y el árbol de diagnóstico para transformadores de potencia como modelos para incrementar la confiabilidad operacional.

\section{ANÁLISIS DE CRITICIDAD}

El análisis de criticidad es una metodología que permite establecer la jerarquía o prioridades de procesos, sistemas y activos creando una estructura que facilite la toma de decisiones acertadas y efectivas, direccionando el esfuerzo y los recursos en áreas donde es más importante y/o necesario mejorar la confiabilidad operacional basada en la realidad actual. El mejoramiento de la confiabilidad operacional de cualquier instalación o de sus sistemas y componente está asociado con cuatro aspectos fundamentales: confiabilidad humana, confiabilidad del proceso, confiabilidad del diseño y la confiabilidad del mantenimiento. (Huerta, 2001).

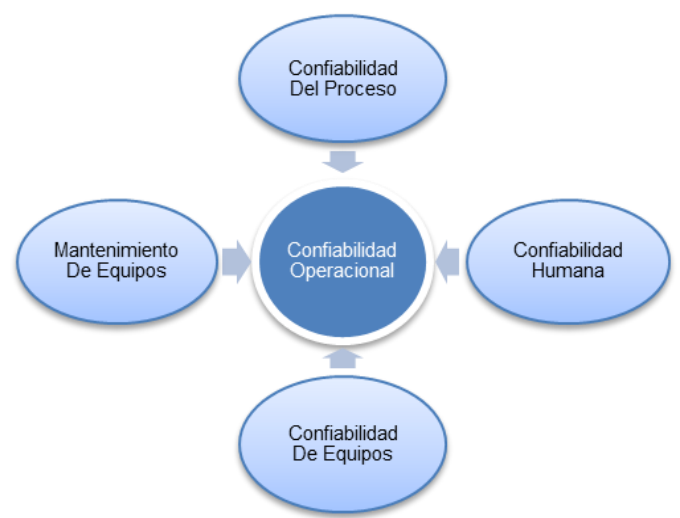

Fig. 1. Aspectos de la confiabilidad operacional.

Los activos a los cuales se les aplicó el análisis de criticidad para su jerarquización se encuentran en el nivel de tensión de $115 \mathrm{KV}$ de la subestación Belén ubicada en zona urbana de la ciudad de Cúcuta, Colombia, siendo un total 57 elementos que están conectados en configuración anillo como se muestra en la figura 2

Para recopilar la información necesaria se aplicaron encuestas a un equipo de trabajo previamente seleccionado, idóneo y altamente capacitado; este grupo de trabajo pertenece al área de mantenimiento de la subgerencia de subestaciones y líneas de Centrales Eléctricas del Norte de Santander SA ESP y está compuesto por: profesional P2 mantenimiento de subestaciones, tecnólogo E mantenimiento de subestaciones, tecnólogo D mantenimiento predictivo, tecnólogo D - expansión y reposición de líneas y tecnólogo $\mathrm{D}$ - protecciones.

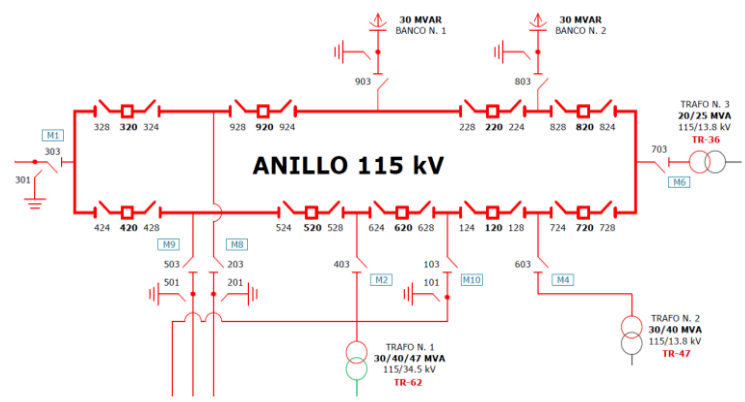

Fig. 2. Diagrama unifilar S/E Belén - $115 \mathrm{KV}$

El esquema de encuesta que se entregó al personal de mantenimiento está compuesto por 7 preguntas, las cuales se ajustaron a las necesidades, políticas y objetivos de calidad de la empresa y en las cuales intervienen los siguientes parámetros:

- Frecuencia de fallas

- Tiempo promedio para reparar

- Impacto sobre la prestación del servicio

- Costo de reparación o reposición

- Impacto en la seguridad personal

- Impacto ambiental

- Impacto satisfacción de clientes internos

Las preguntas tienen diferente ponderación, la cual depende de la jerarquización que sugirió el ingeniero jefe de mantenimiento de subestaciones. Luego de obtener la totalidad de encuestas para los activos, se procede a tabular los datos obtenidos y posteriormente calcular los valores cuantitativos de criticidad para cada uno de los 57 activos.

Para obtener los valores cuantitativos de criticidad se hizo uso de la ecuación (1), en la cual están presentes los diferentes parámetros inmersos dentro de la encuesta aplicada. Al tabular las respuestas de las encuestas, se promedian las ponderaciones de cada parámetro para cada activo, estos valores promedio se ingresan dentro de la ecuación, obteniéndose el valor de criticidad del activo, este valor se aproxima al entero más cercano. La lista jerarquizada se encuentra en la tabla 1.

$C R I T I C I D A D=$ Frecuencia $\cdot$ Consecuencia 
Dónde:

Consecuencia $=\mathrm{a}+\mathrm{b}$

$\mathrm{a}=$ impacto seguridad personal + impacto ambiental + costo reparación + impacto satisfacción clientes internos

$\mathrm{b}=$ impacto en la prestación del servicio $\times$ Tiempo promedio para reparar (Huerta, 2001).

Cada activo se ubica dentro de la matriz de criticidad en donde se cruzan los parámetros globales que intervienen en la determinación de la criticidad: frecuencia y consecuencia, de esta manera, se puede determinar el nivel de criticidad que tiene cada activo dentro del grupo estudiado y su valor cualitativo asociado.

\section{Tabla 1. Jerarquización de activos críticos, subestación belén / $115 \mathrm{KV}$ CENTRALES ELECTRICAS DEL NORTE DE} SANTANDER ANALISIS DE CRITICIDAD

\begin{tabular}{|c|c|c|c|c|c|}
\hline \multicolumn{6}{|c|}{ Subestación Belén / 115KV } \\
\hline Pos. & Activo & Criticidad & Pos. & Activo & Criticidad \\
\hline 1 & TR-36 & 57 & 30 & SA928 & 30 \\
\hline 2 & TR-47 & 57 & 31 & ST603 & 26 \\
\hline 3 & TR-62 & 57 & 32 & ST403 & 26 \\
\hline 4 & IA720 & 54 & 33 & ST303 & 26 \\
\hline 5 & TI-720 & 52 & 34 & SL503 & 26 \\
\hline 6 & IA920 & 48 & 35 & SL203 & 26 \\
\hline 7 & IA820 & 48 & 36 & SL103 & 26 \\
\hline 8 & IA620 & 48 & 37 & SA924 & 26 \\
\hline 9 & IA520 & 48 & 38 & SA828 & 26 \\
\hline 10 & IA420 & 48 & 39 & SA824 & 26 \\
\hline 11 & IA320 & 48 & 40 & SA728 & 26 \\
\hline 12 & IA220 & 48 & 41 & SA724 & 26 \\
\hline 13 & IA120 & 48 & 42 & SA628 & 26 \\
\hline 14 & TI-920 & 45 & 43 & SA624 & 26 \\
\hline 15 & FP420 & 44 & 44 & SA528 & 26 \\
\hline 16 & FP203 & 42 & 45 & SA524 & 26 \\
\hline 17 & FP620 & 40 & 46 & SA428 & 26 \\
\hline 18 & FP320 & 40 & 47 & SA424 & 26 \\
\hline 19 & FP303 & 40 & 48 & SA328 & 26 \\
\hline 20 & TU-920 & 35 & 49 & SA324 & 26 \\
\hline 21 & TU-620 & 35 & 50 & SA228 & 26 \\
\hline 22 & TU-420 & 35 & 51 & SA224 & 26 \\
\hline 23 & TU-320 & 35 & 52 & SA128 & 26 \\
\hline 24 & TI-620 & 35 & 53 & SA124 & 26 \\
\hline 25 & TI- 520 & 35 & 54 & $\mathrm{~S} 903$ & 26 \\
\hline 26 & TI-420 & 35 & 55 & 5803 & 26 \\
\hline 27 & TI-320 & 35 & 56 & M. Y C. & 21 \\
\hline 28 & TI- 120 & 35 & 57 & M. yP. & 20 \\
\hline 29 & ST703 & 30 & & & \\
\hline
\end{tabular}

La determinación de los niveles de criticidad se da de la siguiente manera: si la criticidad de un activo es menor al $25 \%$ del valor máximo posible de criticidad, este activo se ubica en criticidad leve; si el valor de criticidad se encuentra entre el $26 \%$ y el $50 \%$ del valor máximo posible, dicho activo se ubica en criticidad moderada; por ultimo si el valor de criticidad del activo se encuentra dentro del $51 \%$ y el $100 \%$ del valor máximo posible, el activo se ubica en criticidad alta; en este caso el valor máximo posible corresponde a 365 puntos. La matriz de criticidad se muestra en la figura 3 donde sus rangos se identifican por medio de colores, criticidad leve de color verde, criticidad moderada de color amarillo y criticidad alta de color rojo; también sobresalen los valores de criticidad obtenidos para los activos en este análisis .
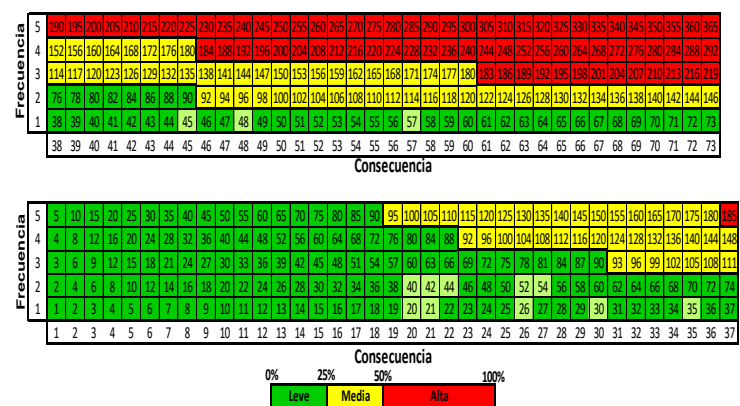

Fig. 3: Matriz de criticidad.

La figura 3 muestra que el grupo de activos analizados está ubicado en su totalidad en el nivel de criticidad leve, lo que evidencia buenas prácticas de mantenimiento en torno a este grupo de activos, afianzando la confiabilidad operacional de los mis mos.

\section{DIAGNÓS TICO DE FALLAS PARA TRANSFORMADORES DE POTENCIA}

La técnica del árbol de fallas, FTA, surgió en 1962 con su primera aplicación a la verificación de la fiabilidad de diseño del cohete Minuteman. Posteriormente ha sido aplicada sobre todo inicialmente en el campo nuclear y posteriormente en el campo químico. (Protección Civil, 2015).

El FTA consta los siguientes pasos:

1. Definir el evento superior o suceso top.

2. Conocer el sistema.

3. Construir el árbol.

4. Validar el árbol.

5. Evaluar el árbol.

6. Considerar cambios constructivos.

7. Considerar alternativas y recomiende medidas.

\subsection{Análisis de Fallas en transformadores de potencia}

Los transformadores al ser máquinas eléctricas estáticas, no poseen partes móviles, por tal motivo no requieren maniobras de mantenimiento con tanta frecuencia como en otros tipos de máquinas eléctricas, lo que no quiere decir que tales maniobras 
no se hagan; se debe resaltar que en la mayoría de los casos las fallas en los transformadores no son hechos espontáneos, estos se dan poco a poco con una serie de eventos o sucesos intermedios; es por esta razón que en la mayoría de los casos donde un transformador se encuentra fuera de servicio, es debido a descuidos en el mantenimiento y en las revisiones periódicas; mediante el presente análisis se pueden determinar las posibles causas y consecuencias que tendrá cada uno de ellos. Para analizar que sucesos pueden ocasionar que un transformador de potencia este fuera de servicio se evaluarán 6 sucesos principales:

1. Disparo en protecciones principales.

2. Incendio o explosión.

3. Sobrecalentamiento

4. Cambiador de tomas fallado.

5. Falla a tierra.

6. Fallas protecciones de respaldo.

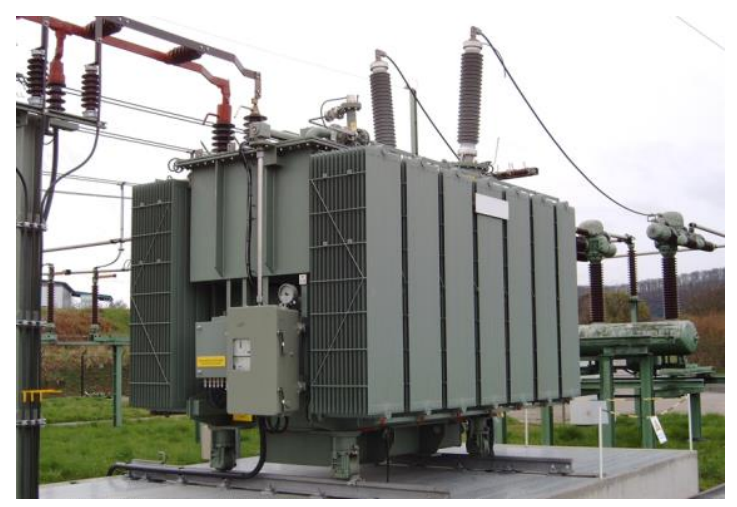

Fig. 4. Transformador de potencia.

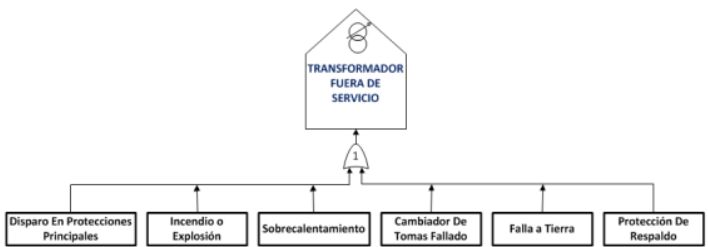

Fig. 5. Árbol de diagnóstico de fallas.

Al usar aceite y papel como aislantes en la construcción de transformadores, se debe tener en cuenta que estos dos componentes envejecen y se descomponen con el tiempo debido a su uso normal, determinando en gran medida la vida útil del transformador.

El calentamiento excesivo junto a la humedad presente en el interior del transformador son los sucesos básicos que mayor incidencia tienen en la falla de un transformador, al intervenir en al menos dos tercios de los eventos principales causantes de un transformador fuera de servicio.

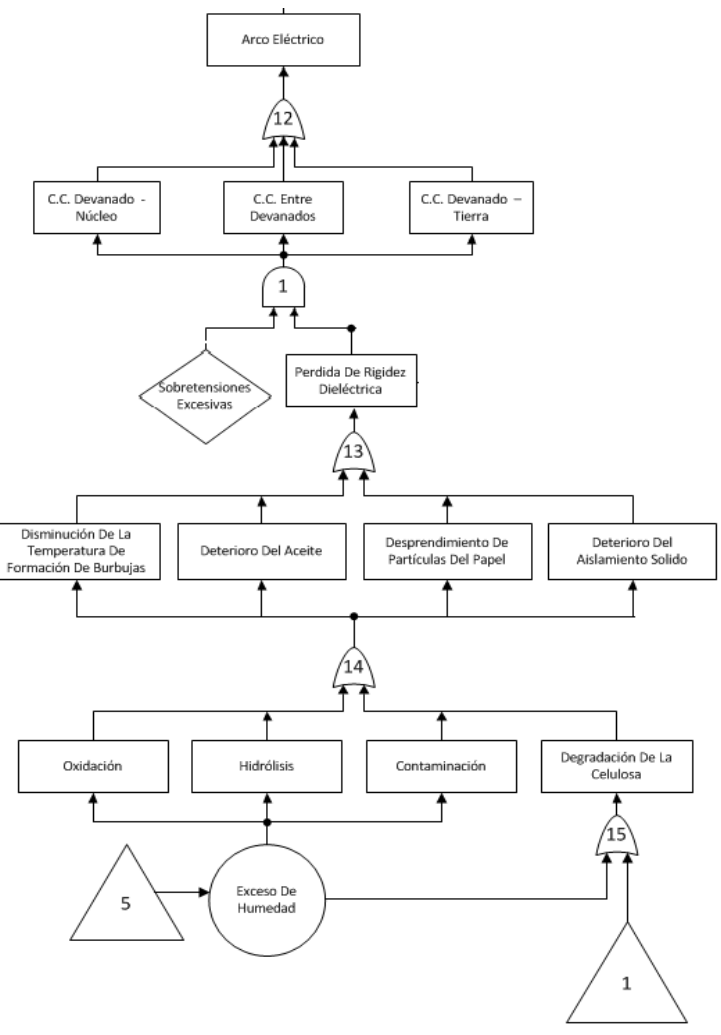

Fig. 6. Construcción del árbol de diagnóstico de fallas (continuación).

La humedad en particular es un parámetro físico de sumo cuidado que se debe medir rigurosamente en el interior del transformador; este parámetro es intrínseco en los materiales aislantes internos del transformador; en el proceso de construcción se somete al trasformador a ciertos procesos de secado para liberar de la humedad al aceite, la madera y el papel entre otros; debemos partir del hecho que no se podrá obtener un transformador con $0 \%$ de humedad, pero en porcentajes pequeños dicho parámetro es permisible.

La presencia de cantidades de humedad por fuera de los parámetros establecidos asociado a la presencia de altas temperaturas dentro de la cuba del transformador genera degradación de la celulosa, componente principal en el papel, que a su vez genera desprendimiento de partículas, también se genera oxidación e hidrolisis en el aceite y contaminación al interior de la cuba; el aceite al perder sus propiedades físico-químicas deteriora el aislamiento sólido del transformador. Los anteriores sucesos causan la perdida de rigidez dieléctrica y más humedad; por ello la humedad debe ser controlada con especial cuidado, ya que la formación de la misma puede convertirse en un proceso cíclico que afecta directamente la vida de los aislamientos internos del transformador, 
reduciendo la vida útil de éste y provocando fallas como la generación de arcos eléctricos, los cuales de no ser detectados y despejados correctamente por las protecciones principales debido a desajustes de parámetros o fallas en cableado, pueden generar altas temperaturas que vaporizan el aceite y originan gases inflamables como el metano $\left(150^{\circ} \mathrm{C}\right)$, etano $\left(1000^{\circ} \mathrm{C}\right)$, etileno $\left(1000^{\circ} \mathrm{C}\right) \mathrm{y}$ acetileno $\left(3000^{\circ} \mathrm{C}\right)$.

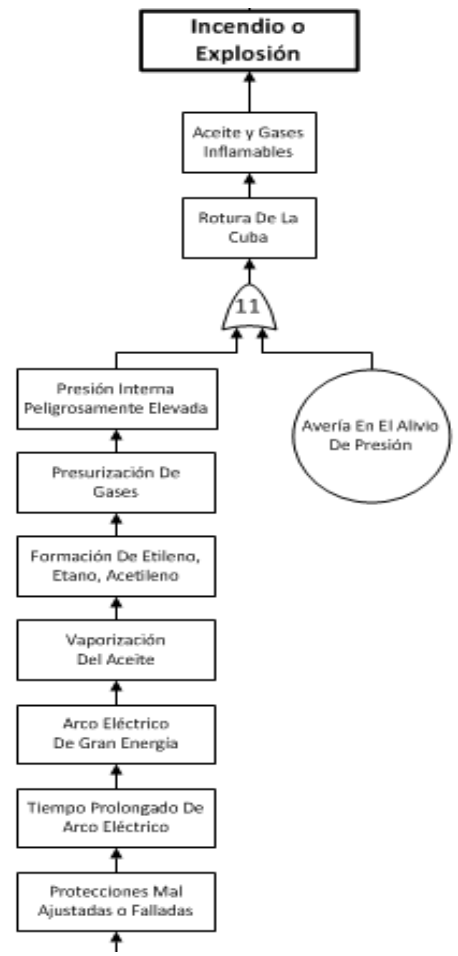

Fig. 7. Construcción del árbol de diagnóstico de fallas (continuación).

Asociado a las altas temperaturas se generan altas presiones al interior de la cuba del transformador, que pueden ocasionar rotura de la cuba e ignición; provocando, además de la salida de servicio del transformador, pérdidas totales en el transformador asociado a graves perjuicios económicos y ambientales.

Por sí solo, el calentamiento excesivo o sobrecalentamiento es un suceso principal que conlleva a que un transformador quede fuera de servicio, el origen del sobrecalentamiento va desde sobrecargas en la red, fallas en la refrigeración forzada, puntos calientes hasta fugas de aceite. Las fallas en la refrigeración son consecuencia directa de averías mecánicas o eléctricas de los ventiladores o las bombas de aceite dependiendo de cuál sea el caso en trasformadores refrigerados ONAF, OFAF $u$ OFAN.

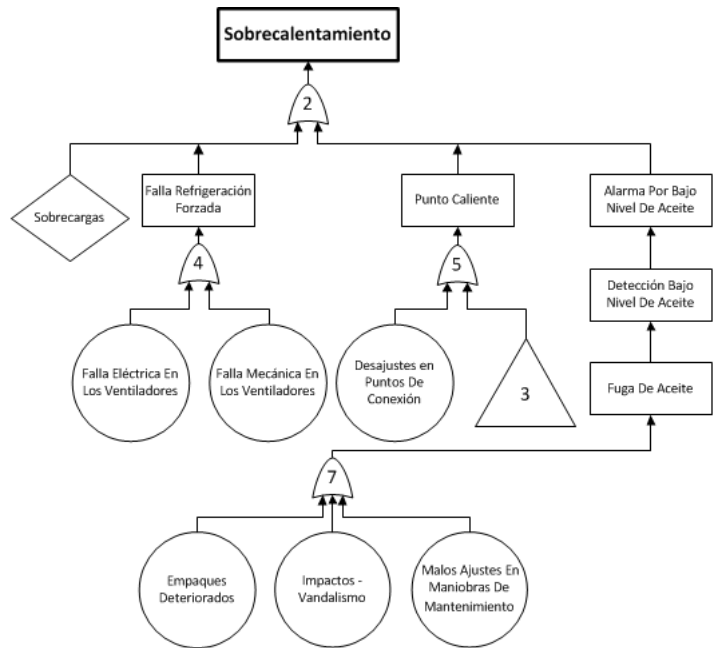

Fig. 8. Construcción del árbol de diagnóstico de fallas (continuación).

Las fugas de aceite pueden ser ocasionadas poractos vandálicos que deforman el transformador, por deterioro de los empaques o por maniobras de mantenimiento mal ejecutadas dejando puntos desajustados. Los puntos calientes se originan debido a malos ajustes en puntos de conexión y cortocircuitos entre espiras; estos puntos calientes generan estrés térmico en el elemento que tiende a fundir el material, debido a la elasticidad del mismo y a no alcanzarse las temperaturas de fusión, el material tiende a cristalizar su estructura química produciendo fragilidad en el mismo y en el caso de esfuerzos dinámicos grandes producidos por cortocircuitos de altas corrientes o ráfagas de viento considerables, el punto caliente tendera a destruirse ocasionando una falla mayor.

Las sobrecargas generan aumentos de temperatura que pueden ser de corta duración, es el caso de la puesta en marcha de grandes industrias que generan altas corrientes de magnetización momentáneas; este aumento de temperatura en el interior del transformador envejece considerablemente los aislamientos internos del mismo y también inciden en la generación de burbujas de gas; las burbujas pueden producirse cuando la temperatura del punto más caliente supera en promedio los $140^{\circ} \mathrm{C}$ para un transformador con un contenido del $2 \%$ de humedad en el aislamiento de los arrollamientos. La temperatura de inicio de formación de burbujas disminuye con el aumento de la concentración de humedad; las burbujas generalmente no se forman en regiones de alto estrés eléctrico, pero si viajan a regiones con alto estrés eléctrico pueden caus ar una reducción significativa de la rigidez dieléctrica. Las sobrecargas también pueden generar aumentos en la temperatura de larga duración al interior de la cuba, 
no son comunes pero de producirse durante un tiempo prolongado pueden afectar considerablemente la vida útil del transformador deteriorando los aislamientos internos aceleradamente y, de igual modo, a los empaques. La resistencia de contacto en el cambiador de tomas en carga puede aumentar con corrientes y temperaturas altas generando en algunos casos problemas térmicos en el mismo volviendo frágiles las uniones del trasformador como resultado de las altas temperaturas. En general, estos aumentos en la temperatura y de las corrientes de los arrollamientos y demás conductores internos del transformador, ocasionan un riesgo prematuro de falla; este riesgo asociado al aumento en la densidad de flujo de dispersión que provoca altas corrientes de Foucault en los conductores y calentamientos en las partes metálicas y en la cuba, al aumento de humedad y contenido de gases en el aislamiento y a la exposición de mayores potenciales en los bornes, cambiadores de tomas y transformadores de corriente tienen como consecuencia daños importantes a corto plazo o puede presentarse un envejecimiento acumulado del activo.

Asimismo, la salida de servicio de un transformador puede originarse por la actuación de las protecciones principales, que son el mejor evento que podría darse para la salida de servicio de un transformador de potencia, ya que protegen al transformador de daños graves cuando se ajustan con el debido cuidado y precisión. La generación de arcos eléctricos al interior de la cuba del transformador, como se explicó anteriormente, tienen su génesis en el aumento no controlado de la humedad y la temperatura, como consecuencia de estos arcos eléctricos se generan grandes presiones dentro de la cuba, en algunos casos momentáneas que causan el movimiento de aceite hacia el deposito conservador a velocidades fuera de lo común; estos hechos provocan la detección y disparo de las protecciones de sobre-flujo, sobrepresión, y presión súbita las cuales son mecánicas. Los arcos eléctricos también crean corrientes diferenciales en los devanados del transformador que son detectadas por el relé diferencial produciendo disparo y desconexión del transformador, la protección diferencial también puede producir un disparo cuando se originan altas corrientes de magnetización que se presenta en uno de los devanados del transformador al momento de la puesta en servicio del mismo, estas corrientes son detectados y despejados por la protección diferencial. Por último también se pueden generar disparos debido a la detección de sobre-flujo magnético a raíz de la disminución de la relación voltaje/frecuencia en la red.

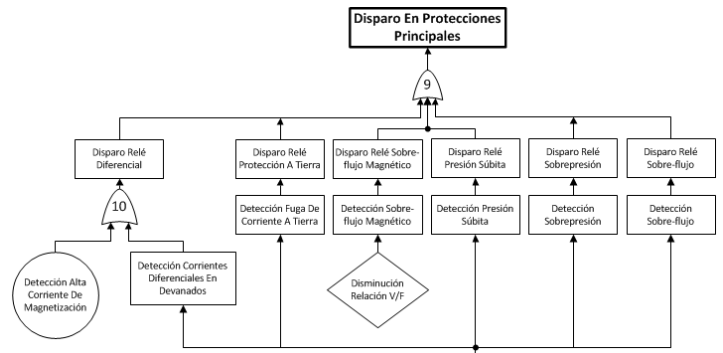

Fig. 9: Construcción del árbol de diagnóstico de fallas (continuación).

Otro de los eventos principales que genera la salida de operación de un transformador es las fallas a tierra, las cuales se generan por bujes fallados o perdidas de rigidez dieléctrica asociada a sobrecargas que dan inicio a arcos eléctricos hacia las partes aterrizadas del transformador. Un buje o bushing puede resultar fallado debido a puntos calientes ocasionados por malos ajustes en labores de mantenimiento, por disminución del aislamiento interno del buje, por presencia de plantas o animales sobre el buje, por deformación del buje por impactos, por vandalismo o por sobreajustes en maniobras de mantenimiento.

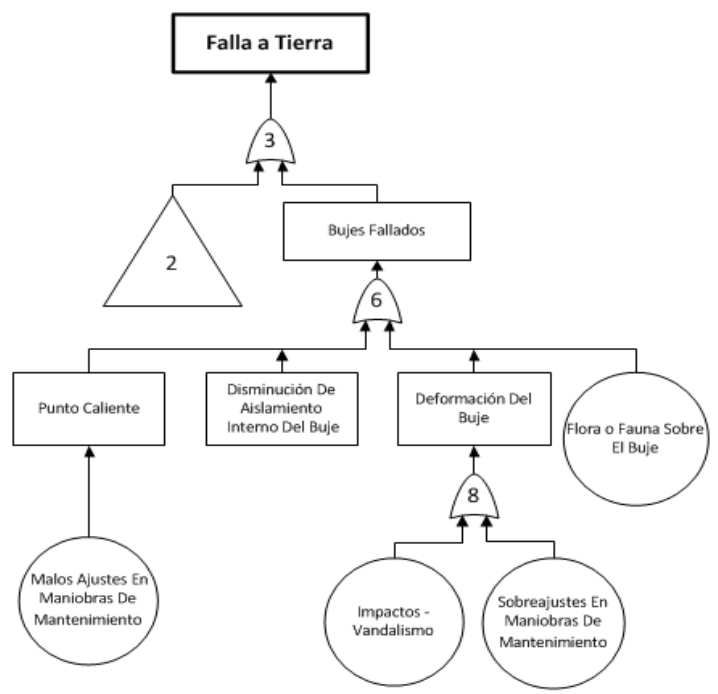

Fig. 10: Construcción del árbol de diagnóstico de fallas (continuación).

El cambiador de tomas es un elemento que también puede sacar de servicio el transformador, en el caso del OLTC (Cambiador de Tomas en Carga) se pueden producir disparo por un bajo nivel de aceite dentro del mismo. Es normal que dentro del OLTC se generen pequeños arcos eléctricos al momento de regular la carga, debido al movimiento de los contactos en el cambio de los taps, por lo cual el OLTC tendrá mayor contaminación de gases y 
burbujas, mayor deterioro del aceite y habrá presencia más alta de humedad con respecto al aceite presente en la cuba del transformador, razón por la cual el aceite del OLTC se separa del aceite al interior de la cuba.

Cuando suceden arcos eléctricos que disipan gran cantidad de energía, estos generan gases y aument os de presión en muy poco tiempo, es allí cuando se ejecutan disparos por presión súbita, sobrepresión o disparos por el relé de Jansen que es un tipo de relé aplicado a los OLTC, dicho relé permite el paso de aceite al depósito de expansión en condiciones normales, cuando se producen cambios bruscos en el flujo de aceite se activa una especie de flotador en su interior que envía una señal al relé auxiliar, provocando la salida del transformador a través de los interruptores; este relé es análogo al relé de Buchholz en la cuba del transformador.

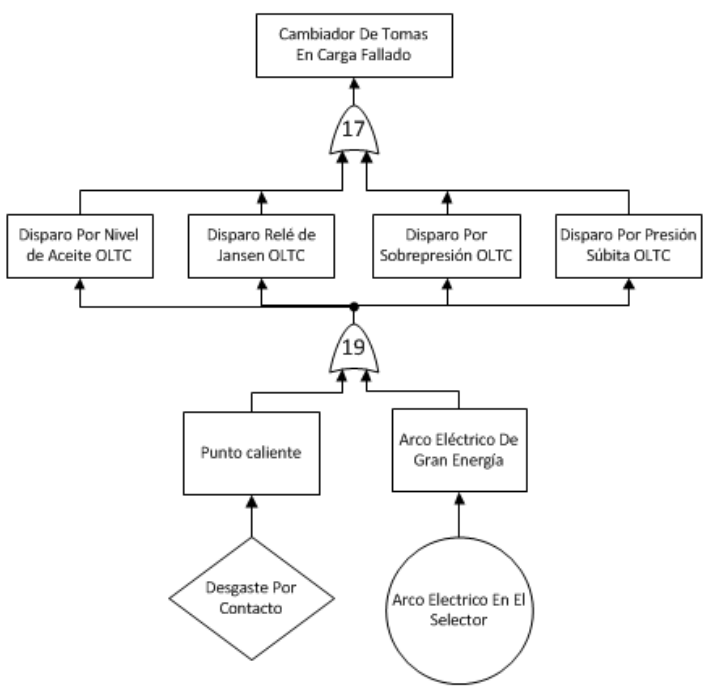

Fig. 11: Construcción del árbol de diagnóstico de fallas (continuación).

Una falla en el cambiador de tomas sin carga puede producirse debido a la mala operación del mismo, deformaciones del mecanismo debido a sobreajustes en la operación, fugas de aceites o por puntos calientes generados por falsos contactos producidos al no quedar el tap en la posición correcta en el momento de un ajuste o mantenimiento, como evidencia se forma carboncillo al rededor del contacto.

Por último se deben tener en cuenta las protecciones de respaldo, las cuales protegen al transformador frente a fallas externas ocurridas en otros subsistemas del sistema de distribución y subtransmisión de CENS S.A. E.S.P., las cuales debido a malos ajustes llegan hasta el transformador.

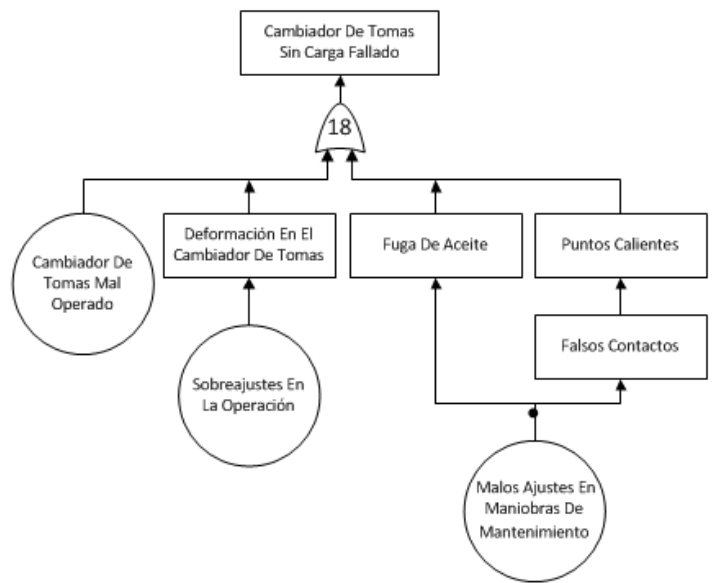

Fig. 12. Construcción del árbol de diagnóstico de fallas (continuación).

La figura 13 muestra el diagrama de árbol completo para el análisis de fallas en transformadores de potencia.

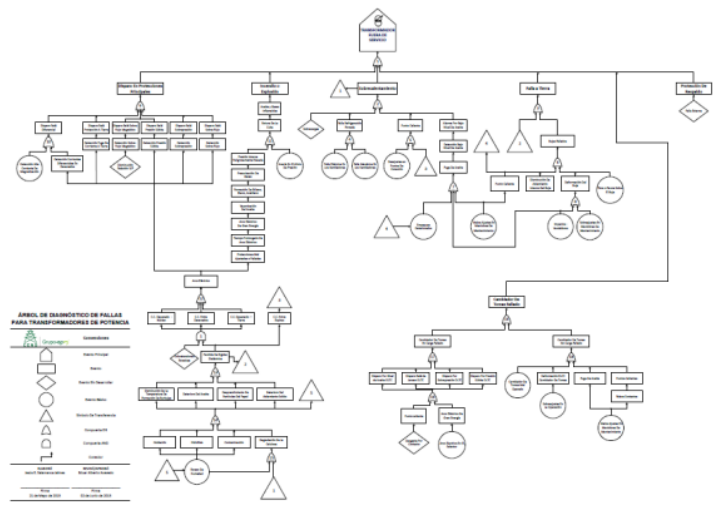

Fig. 13. Árbol de diagnóstico de fallas para transformadores de potencia.

\section{CONCLUS IONES}

Por medio del análisis de criticidad se concluyó que los activo más crítico en el anillo de $115 \mathrm{KV}$ de la subestación Belén, fueron los transformadores de potencia asociados a este nivel de tensión; cuestión que era previsible por la capacidad de potencia, costo y función dentro del sistema; estos transformadores afectan a gran cantidad de usuarios finales de CENS S.A. E.S.P.

La ubicación de los activos dentro de la matriz de criticidad evidencia la correcta planeación y las buenas prácticas de mantenimiento que la subgerencia de subestaciones y líneas promueve para este grupo de activos, teniendo en cuenta que todos los activos se ubicaron en la zona de criticidad baja dentro de la matriz de criticidad. 
Por medio del análisis del árbol de diagnóstico de fallas se concluye que los sucesos básicos que mayor incidencia tienen en ocasionar un transformador fuera de servicio son el aumento de la humedad y de la temperatura, los cuales afectan dos tercios de los eventos principales en el árbol de diagnóstico de fallas para transformadores de potencia.

\section{RECONOCIMIENTO}

Los autores agradecen a Centrales Eléctricas del Norte de Santander SA ESP por la financiación del proyecto.

\section{REFERENCIAS}

Becerra S., G. y García P., O. (2005). Sistema Integrado de Confiabilidad Operacional para el área de servicios industriales de Bavaria $S$. A. Cervecería de Boyacá, Universidad Pedagógica y Tecnológica de Colombia. Tunja, Colombia.
Huerta M., R. (2001). El análisis de criticidad, una metodología para mejorar la confiabilidad operacional, Revista club del mantenimiento, Vol. 6, Buenos Aires, Argentina.

Protección Civil. (2015). Determinación de frecuencias de sucesos complejos, método del árbol de fallos. Guía técnica, Madrid, España. http://www.proteccioncivil.es/catalogo/carpeta02 /carpeta22/guiatec/Metodos_cuantitativos/cuant_ 25.htm (14 de octubre de 2015).

Tarantino A., R.; Aranguren Z., S. (2006). Criterios $\mathrm{y}$ lineamientos introductorios para el desarrollo y aplicación de sistemas de detección y diagnóstico de fallas. Revista Colombiana de Tecnologías de Avanzada, Vol. 2, No. 8.

Tarantino A., R.; Aranguren Z., S. (2006). Diseño de un plan de mantenimiento centrado en confiabilidad: una aplicación industrial. Revista Colombiana de Tecnologías de Avanzada, Vol. 1, No. 7.

Tarantino A., R.; Aranguren Z., S. (2004). Sistemas para la detección y diagnóstico de fallas: implantación industrial. Revista Colombiana de Tecnologías de Avanzada, Vol. 1, No. 3. 